\title{
BOUNDARY CONDITIONS FOR FINITE ELEMENT SIMULATIONS OF CONVECTIVE FLOWS WITH ARTIFICIAL BOUNDARIES
}

\author{
J. C. HEINRICH \\ Department of Aerospace and Mechanical Engineering, The University of Arizona, Tucson, Arizona, 85721, U.S.A. \\ S. R. IDELSOHN \\ Mechanics Laboratory of INTEC, Universidad Nacional del Litoral and Consejo Nacional de Investigaciones \\ Centificasy Técnicas, Sante Fé, Argentina \\ E. OÑATE \\ International Center for Numerical Methods in Engineering, Polytechnic University of Catalunya, Barcelona, Spain \\ C. A. VIONNET \\ Department of Aerospace and Mechanical Engineering, University of Arizona, Tucson, Arizona, 85721, U.S.A.
}

\begin{abstract}
SUMMARY
We examine the use of natural boundary conditions and conditions of the Sommerfeld type for finite element simulations of convective transport in viscous incompressible flows. We show that natural boundary conditions are superior in the sense that they always provide a correct boundary condition, as opposed to the Sommerfeld-type conditions, which can lead to a singular formulation and a great loss of accuracy. For the Navier-Stokes equations, the natural boundary conditions must be combined with a simple method to eliminate perturbations on the pressure at the open boundary, which is the source of most errors.
\end{abstract}

KEY WORDS: finite elements; open boundaries; fluid flow; natural boundary conditions; Sommerfeld radiation condition; Navier-Stokes equations; incompressible flow

\section{INTRODUCTION}

In the numerical simulation of transport phenomena, one must frequently truncate the domain of definition of a physical process, thus introducing one or more artificial boundaries in the computational domain. In order to eliminate reflections in hyperbolic problems, the artificial finite boundaries must absorb all outgoing waves, which is difficult to achieve and has led to a great amount of research on the subject. ${ }^{1-5}$ One of the most widely used absorbing boundary conditions is the Sommerfeld radiation condition, ${ }^{6}$ which is an effective and simple way to treat such open boundaries when applied in the way proposed by Orlanski. ${ }^{7}$

The difficulty in elliptic/parabolic problems, such as viscous flows and convective transport, is that a boundary condition must be applied over the open portion of the boundary in order for the problem to be well posed. Three basic ways to achieve this have been proposed:

1. If the asymptotic behaviour of the solution is known, a Dirichlet boundary condition reflecting such behaviour may be imposed. Often, however, it is not possible to determine in 
advance how far the domain must extend for the solution to achieve the required accuracy. Moreover, such analytical solutions are not available for most practical cases.

2. In most simulations, we resort to the fact that the derivatives should decay to zero 'away from the action', and homogeneous natural boundary conditions are imposed. However, in simulations involving viscous incompressible flows, it has been observed that these boundary conditions may introduce significant perturbations at the open boundary, especially if the flow is stratified. ${ }^{8,9}$ We will examine these boundary conditions and show that they are appropriate under all circumstances, provided the pressure is treated properly at the boundary.

3. Recently, an open boundary condition was proposed by Papanastasiou et al. ${ }^{10}$ in which the contributions of the surface integrals obtained after application of the Gauss divergence theorem to the governing equations are also discretized and retained in the finite element matrices. It was pointed out by Heinrich and Vionnet ${ }^{11}$ that this is equivalent to imposing a Sommerfeld condition when linear elements are used in one dimension and a slightly modified form of the Sommerfeld condition when bilinear or trilinear elements are used in higher dimensions. Moreover, it leads to a singular problem if the convective velocities vanish. We will show that this boundary condition has been successful in modelling incompressible flows because it eliminates the perturbation of the pressure at the boundary. However, it does not offer further advantages over natural boundary conditions. Han et al. ${ }^{12}$ applied Orlanski's open boundary condition to incompressible stratified flows using a finite difference discretization. They also concluded that the success of the method depends on how the pressure is treated at the boundary.

Various ways of dealing with the open boundary conditions hav element literature. ${ }^{8-10,13}$ However, the arguments put forth to ad hoc. It is our intent to analyse and quantify the effect of the boundary conditions on open boundaries. Moreover, we establish conditions that, when properly applied, provide approxima=

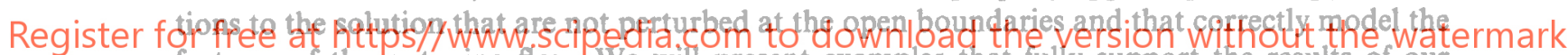
features of the outgoing flow. We will present examples that fully support the results of our analyses.

\section{ONE-DIMENSIONAL CONVECTIVE TRANSPORT}

We will assume from here on that analytic results showing the behaviour of the solution at infinity are not available. We turn our attention to the second and third cases given above.

Consider the steady-state convective diffusion equation

$$
u \frac{\mathrm{d} \phi}{\mathrm{d} x}-\frac{\mathrm{d}}{\mathrm{d} x} D \frac{\mathrm{d} \phi}{\mathrm{d} x}=f, \quad 0 \leqslant x<\infty
$$

with boundary conditions

$$
\phi(0)=\phi_{0}
$$

and

$$
\lim _{x \rightarrow \infty} \phi(x)=K
$$

where $\phi$ is the unknown function, $u$ is the convective velocity, $D$ is the diffusivity, $K$ is a constant (unknown), and $f(x)$ is a given function of $x$ such that $\int_{0}^{\infty} f(x) \mathrm{d} x$ exists. 
The numerical solution of equation (1) using the finite element method requires that the interval $0 \leqslant x<\infty$ be truncated at some finite location, $x=L$, and that a boundary condition be imposed at this point. This is required because the solution depends on two constants; hence, only one boundary condition would lead to an undetermined problem with infinitely many solutions. Therefore, any numerical method that finds one of these solutions will do so because a second boundary condition has been applied at $x=L$, even though the exact nature of the second boundary condition may be not be immediately clear.

The weak form of equation (1) over the interval $0 \leqslant x \leqslant L$ is

$$
\int_{0}^{L}\left(D \frac{\mathrm{d} w}{\mathrm{~d} x} \frac{\mathrm{d} \phi}{\mathrm{d} x}+u w \frac{\mathrm{d} \phi}{\mathrm{d} x}-w f\right) \mathrm{d} x-\left.\left(w D \frac{\mathrm{d} \phi}{\mathrm{d} x}\right)\right|_{x=L}=0
$$

where $w$ is an admissible weighting function.

From the boundary condition an infinity, equation (3), it follows that if $L$ is large enough, imposing the natural boundary condition

$$
\left.D \frac{\mathrm{d} \phi}{\mathrm{d} x}\right|_{x=L}=0
$$

should provide a good approximation to the solution, and we will see that this is indeed the case. It also follows that the boundany termat $x=4$ must vanish and not contribute to the system of equations.

On the other hand, i using the shape functions and is incorporated into the last ro apparently would be equivalent to not imposing a boundary condition a $x$ be the case in a boundary value problem. It was shown in Reference 11 that, for the timedependent case, with $f(x) \equiv 0$, discretizing the boundary term is equivalent to imposing the

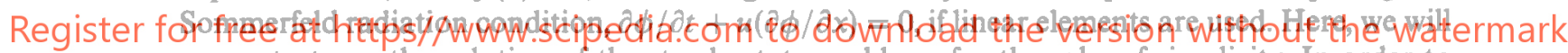
concentrate on the solution of the steady-state problems for the sake of simplicity. In order to understand the resulting boundary condition, we must examine each element type individually.

We will do this for linear and quadratic elements.

\subsection{Linear elements}

We now assume that the interval $0 \leqslant x \leqslant L$ has been discretized using linear elements of size $h$. We look at the element equations for the last element, $L-h \leqslant x \leqslant L$, with nodes $x_{n}=L-h$ and $x_{n+1}=L$. Assuming constant $D$ and $u$, the Galerkin form of equation (4) yields

$$
\left\{\frac{D}{h}\left[\begin{array}{cc}
1 & -1 \\
-1 & 1
\end{array}\right]+\frac{u}{2}\left[\begin{array}{cc}
1 & -1 \\
-1 & 1
\end{array}\right]\right\}\left[\begin{array}{c}
\phi_{n} \\
\phi_{n+1}
\end{array}\right]=\left[\begin{array}{c}
\bar{f}_{n} \\
\bar{f}_{n+1}
\end{array}\right]+\left[\begin{array}{c}
0 \\
\left.D \frac{\mathrm{d} \phi}{\mathrm{d} x}\right|_{x=L}
\end{array}\right]
$$

where

$$
\bar{f}_{k}=\int_{L-h}^{L} N_{k}(x) f(x) \mathrm{d} x, \quad k=n, n+1
$$

and $N_{k}$ are the linear shape functions. 
If the natural boundary condition (5) is applied at $x=L$, the last equation in the linear system takes the form

$$
\frac{D}{h}\left(\phi_{n+1}-\phi_{n}\right)+\frac{u}{2}\left(\phi_{n+1}-\phi_{n}\right)=\bar{f}_{n+1}
$$

which yields equation (5) in the limit as $h \rightarrow 0$.

If the boundary term in equation (6) is discretized and retained as proposed in Reference 10, we obtain

$$
\left\{\frac{D}{h}\left[\begin{array}{rr}
1 & -1 \\
0 & 0
\end{array}\right]+\frac{u}{2}\left[\begin{array}{ll}
-1 & 1 \\
-1 & 1
\end{array}\right]\right\}\left[\begin{array}{c}
\phi_{n} \\
\phi_{n+1}
\end{array}\right]=\left[\begin{array}{c}
\bar{f}_{n} \\
\bar{f}_{n+1}
\end{array}\right]
$$

Taking the limit as $h \rightarrow 0$, the last equation yields

$$
\left.u \frac{\mathrm{d} \phi}{\mathrm{d} x}\right|_{x=L}=f(L)
$$

This is the boundary condition applied when the boundary term is retained in the finite element formulation, and it can be easily verified through examples. It also follows that the problem is well posed only if $u \neq 0$. If $\boldsymbol{u}=0$, a singular global stifiness matrix results. It is also easily verified that retaining the boundary term in equation (6) is equivalent to assuming that, at $x=L$, we have an interface with an inviscid (non-diffusive) mediun for $x>L$. For these reasons, we will refer to it the "convection boundary condition'.
We now illustrate the effect of the boundary conditions through an example equation (1) with $D=1, \phi_{0}=0$, and $f(x)=$ parameter. The exact solution is then given by

Register for free at https//www.scipedia.com to download the version without the watermark

$$
\phi(x)=\frac{1-\mathrm{e}^{-x}}{1+u}
$$

We use $h=0.05$ in all cases and calculate with $L=1 \cdot 0,2 \cdot 0$ and 4.0 and with $u=0 \cdot 1,1.0$ and 10.0 in order to study the effect of the location of $L$ and of the convective velocity $u$.

Table I shows the maximum error (at the node $x=L$ ), which shows the expected behaviour: as $L$ and/or $u$ increase, the error decreases accordingly. Also, as the convective velocity $u$ becomes small, the singularity in the convection boundary condition becomes evident, while the natural boundary condition leads to small errors. However, for higher values of $u$, the convection boundary condition is better. The solution using the convection boundary condition always yields an upper bound to the exact solution, while the natural boundary condition gives a lower bound. This is illustrated in Figure 1 for the case $L=u=1 \cdot 0$. The average of the two solutions is an excellent approximation to the exact one, and this should be the case in a wide variety of situations. The method could be used to improve approximations in the absence of other information.

For the examples presented here, it is clear that the convection boundary condition will, in general, produce solutions greater than those obtained using the natural boundary condition, because a positive gradient is enforced at the outflow boundary. The reasons for the convection boundary condition producing solutions greater than the exact solution are, however, not so obvious. 
Table I. Relative error (in per cent) at $x=L$ in the solution of equation (1) with $D=1$ and $f(x)=\mathrm{e}^{-x}$, using linear elements with natural and convection boundary conditions at $x=L$

\begin{tabular}{|c|c|c|c|c|c|c|}
\hline \multirow[b]{2}{*}{$L^{u}$} & \multicolumn{3}{|c|}{$\begin{array}{l}\text { Convection boundary } \\
\text { condition }\end{array}$} & \multicolumn{3}{|c|}{$\begin{array}{l}\text { Natural boundary } \\
\text { condition }\end{array}$} \\
\hline & 0.1 & 1.0 & 10.0 & 0.1 & 1.0 & 10.0 \\
\hline $\begin{array}{l}1.0 \\
2.0 \\
4.0\end{array}$ & $\begin{array}{r}564 \\
289 \\
63\end{array}$ & $\begin{array}{r}38 \\
14 \\
2\end{array}$ & $\begin{array}{l}0.700 \\
0.200 \\
0.003\end{array}$ & $\begin{array}{r}55 \\
28 \\
6\end{array}$ & $\begin{array}{r}37 \\
14 \\
2\end{array}$ & $\begin{array}{l}6.0 \\
2.0 \\
0.2\end{array}$ \\
\hline
\end{tabular}
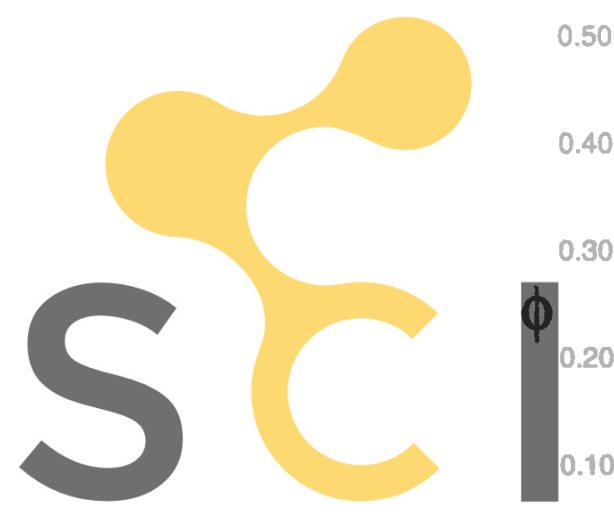

\section{exact}

\section{convection b.c.} natural b.c.
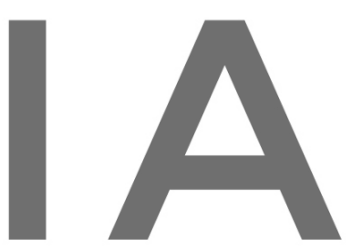

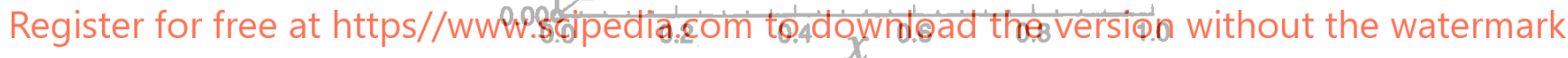
Figure 1. Analytical and aumerical solutions to equation (1) with $D=u=L=1 \cdot 0, f(x)=e^{-x}$, and $\phi_{0}=0$, using 20
linear elements

\subsection{Quadratic elements}

We assume that the elements have size $h$ and that the last element has nodes located at $x_{n-1}=L-h, x_{n}=L-h / 2$, and $x_{n+1}=L$. The Galerkin discretization of equation. (4) yields

$$
\begin{gathered}
\left\{\frac{D}{6 h}\left[\begin{array}{rrr}
14 & -16 & 2 \\
-16 & 32 & -16 \\
2 & -16 & 14
\end{array}\right]+\frac{u}{6}\left[\begin{array}{rrr}
-3 & 4 & -1 \\
-4 & 0 & 4 \\
1 & -4 & 3
\end{array}\right]\right\}\left[\begin{array}{c}
\phi_{n-1} \\
\phi_{n} \\
\phi_{n+1}
\end{array}\right] \\
=\left[\begin{array}{c}
\bar{f}_{n-1} \\
\bar{f}_{n} \\
\bar{f}_{n+1}
\end{array}\right]+\left[\begin{array}{c}
0 \\
0 \\
\left.D \frac{\mathrm{d} \phi}{\mathrm{d} x}\right|_{x=L}
\end{array}\right]
\end{gathered}
$$


Application of the natural boundary condition at $x=L$ leads to the same behaviour as observed for linear elements; therefore, we do not need to discuss this further. The convection boundary condition, on the other hand, produces the relation

$$
\left\{\frac{D}{6 h}\left[\begin{array}{rrr}
14 & -16 & 2 \\
-16 & 32 & -16 \\
-4 & 8 & -4
\end{array}\right]+\frac{u}{6}\left[\begin{array}{rrr}
-3 & 4 & -1 \\
-4 & 0 & 4 \\
1 & -4 & 3
\end{array}\right]\right\}\left[\begin{array}{c}
\phi_{n-1} \\
\phi_{n} \\
\phi_{n+1}
\end{array}\right]=\left[\begin{array}{c}
\bar{f}_{n-1} \\
\bar{f}_{n} \\
\bar{f}_{n+1}
\end{array}\right]
$$

for the last element.

In this case, the diffusion term does not vanish in the equation for the last node; however, two linearly dependent expressions are obtained in the second and third rows of the diffusion matrix that reflect the singularity of the system in the absence of convection, i.e., when $u=0$. A Taylor series analysis shows that the third difference equation in equation (12) leads to

$$
\left.\left(-\frac{\mathrm{d}}{\mathrm{d} x} D \frac{\mathrm{d} \phi}{\mathrm{d} x}+u \frac{\mathrm{d} \phi}{\mathrm{d} x}\right)\right|_{x=L}=f(L)+O(h)
$$

where the diffusion term is in fact a central approximation to the second derivative at node $x_{n}$. This term introduces a perturbation at the boundary and induces oscillations in the solution unless the problem is highly dominated by convection.

To illustrate the behaviour of the quadratic elements with a convection boundary condition, we apply it to the example of the previous section, satting $h=0$ ) 1 so that the same number of nodes is used as in the case of linear elements. When $u=01$, the results are disastrous. The solutions ate useless as they are negative throughout the domain and are two orders of magnitucle larger id absolute value than the exact solution. For the case $u=1 \cdot 0$, the resulting magnitude to those obtained using linear elements, but the solutions are oscillatory smooth and accurate approxinations are obtained, with naxinum errers at $x=1$ of $0.00,0.02$ and 0.003 per cent for $L=1 \cdot 0,2 \cdot 0$, and $4 \cdot 0$, respectively.

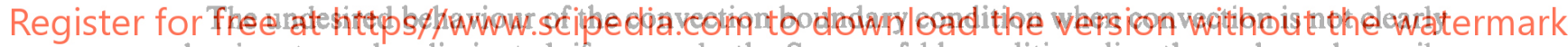
dominant can be eliminated, if we apply the Sommerfeld condition directly, and can be easily achieved, setting the diffusion term for the boundary element to zero. This is accomplished by replacing the diffusion matrix in equation (12) with

$$
\frac{D}{6 h}\left[\begin{array}{rrr}
14 & -16 & 2 \\
-16 & 32 & -16 \\
0 & 0 & 0
\end{array}\right]
$$

which is equivalent to applying the boundary condition, equation (9), at $x=L$ in the limit as $h \rightarrow 0$. The use of this boundary condition with quadratic elements yields practically the same results as using linear elements with the convection boundary condition at $x=L$. In particular, for our example with $D=1$ and $f(x)=\mathrm{e}^{-x}$, we obtained the same errors as shown in the left half of Table I, with the exception of the entry for $L=4 \cdot 0$ and $u=10 \cdot 0$, where quadratic elements with the Sommerfeld boundary condition implemented in the way shown above produced a larger error of 0.02 per cent. In fact, it is interesting to note that the use of this boundary condition did not result in better accuracy anywhere in the domain when compared with the solution using linear elements. On the other hand, quadratic elements in conjunction with the convection boundary condition performed very poorly when convection was not dominant.

We will terminate our discussion of quadratic elements here, and will concentrate on the use of bilinear and trilinear elements in two and three dimensions. 


\section{CONVECTIVE TRANSPORT IN HIGHER DIMENSIONS}

We now examine the extension of the above results to two-dimensional equations* of the form

$$
u \frac{\partial \phi}{\partial x}+v \frac{\partial \phi}{\partial y}-\frac{\partial}{\partial x} D \frac{\partial \phi}{\partial x}-\frac{\partial}{\partial y} D \frac{\partial \phi}{\partial y}=f(x, y)
$$

We will assume that components $u$ and $v$ of the convective velocity and the diffusion coefficient $D$ are constant in order to make the integrations possible. Furthermore, without loss of generality, we will assume that equation (13) is defined over a rectangular domain $\Omega$ given by $0<x<a$ and $0<y<b$, where the boundary $x=a$ is an artificial, open boundary. Let us consider a bilinear element adjacent to the artificial boundary, as shown in Figure 2.

The weak form of equation (14), retaining the line integral along the boundary $x=a$, is

$$
\int_{\Omega}\left\{D\left(\frac{\partial w}{\partial x} \frac{\partial \phi}{\partial x}+\frac{\partial w}{\partial y} \frac{\partial \phi}{\partial y}\right)+w\left(u \frac{\partial \phi}{\partial x}+v \frac{\partial \phi}{\partial y}-f\right)\right\} \mathrm{d} \Omega-\int_{0}^{b} D w \frac{\partial \phi}{\partial x} \mathrm{~d} y=0
$$

Utilizing bilinear elements, the Galerkin equations for an element $e$ adjacent to the artificial boundary as shown in Figure 2 are
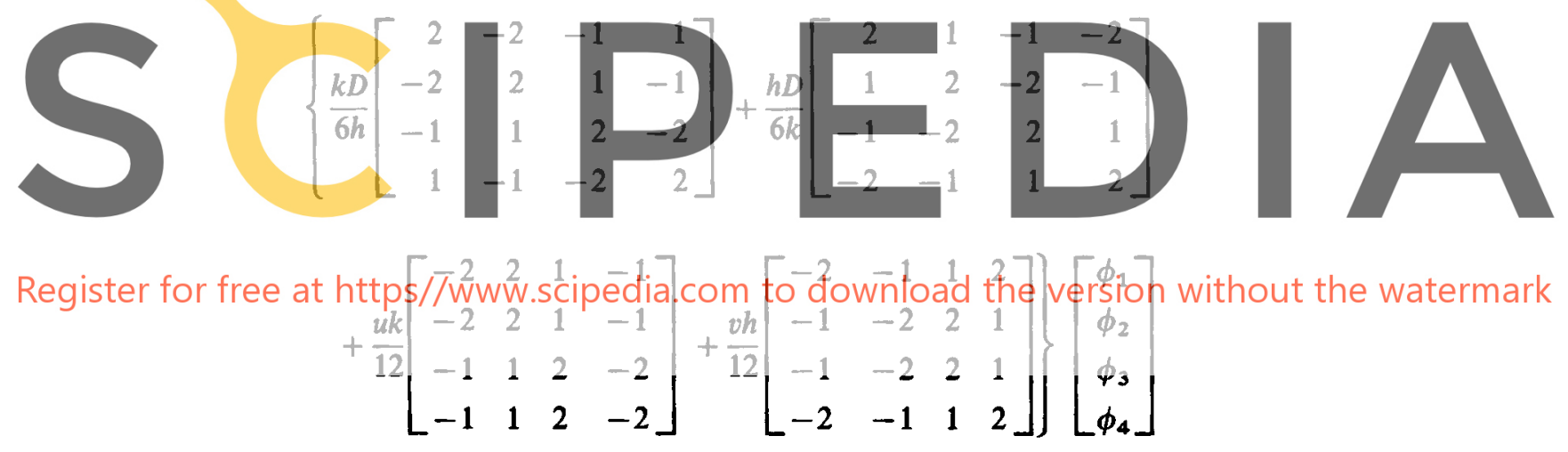

$$
=\left[\begin{array}{c}
\bar{f}_{1} \\
\bar{f}_{2} \\
\bar{f}_{3} \\
\bar{f}_{4}
\end{array}\right]+\left[\begin{array}{c}
0 \\
\int_{0}^{b} D\left(1-\frac{y}{k}\right) \frac{\partial \phi}{\partial x} \mathrm{~d} y \\
\int_{0}^{b} D\left(\frac{y}{k}\right) \frac{\partial \phi}{\partial x} \mathrm{~d} y \\
0
\end{array}\right]
$$

where $\bar{f}_{i}=\int_{e} N_{i} f \mathrm{~d} \Omega$, as in equation (7), with $N_{i}(x, y)$ the bilinear shape functions.

\footnotetext{
* All the results presented in this paper for two dimensions are also valid for three-dimensional equations when trilinear elements are used
} 


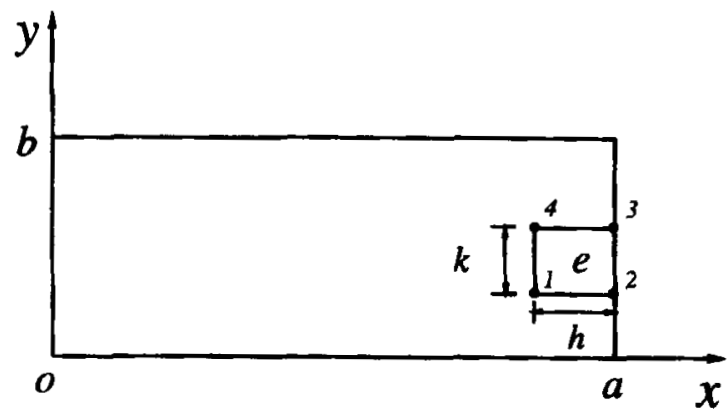

Figure 2. Rectangular two-dimensional domain with a bilinear element adjacent to the artificial boundary $x=a$

Imposing the natural boundary condition $\left.D(\partial \phi / \partial x)\right|_{x=a}=0$ eliminates the second term on the right-hand side and yields equations of the form

$$
\left[-D \frac{\partial \phi}{\partial x}+\frac{h}{2}\left(u \frac{\partial \phi}{\partial x}+v \frac{\partial \phi}{\partial y}-\frac{\partial}{\partial y} D \frac{\partial \phi}{\partial y}\right)\right]_{x=a}=0
$$

at the boundary nodes. Therefore, as $h \rightarrow 0$, the natural boundary condition is satisfied.

The convection boundary condition, on the other hand, produces the element equations
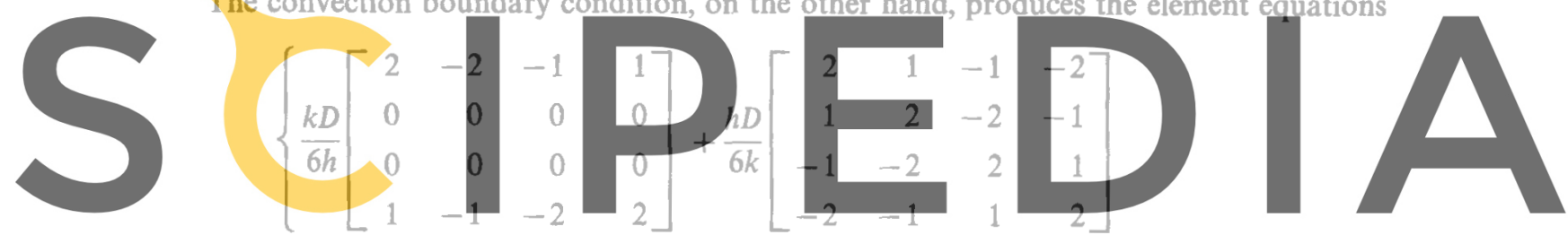

Register for free at https//www.scipedia.com to download the version without the watermark

$$
\left.+\frac{u k}{\mathbf{1 2}}\left[\begin{array}{rrrr}
-2 & 2 & 1 & -1 \\
-2 & 2 & 1 & -1 \\
-1 & 1 & 2 & -2 \\
-1 & 1 & 2 & -2
\end{array}\right]+\frac{v k}{12}\left[\begin{array}{cccc}
-2 & -1 & 1 & 2 \\
-1 & -2 & 2 & 1 \\
-1 & -2 & 2 & 1 \\
-2 & -1 & 1 & 2
\end{array}\right]\right\}\left[\begin{array}{l}
\phi_{1} \\
\phi_{2} \\
\phi_{3} \\
\phi_{4}
\end{array}\right]=\left[\begin{array}{l}
f_{1} \\
\overline{f_{2}} \\
\overline{f_{3}} \\
\overline{f_{4}}
\end{array}\right]
$$

and the nodes on the artificial boundary satisfy the boundary condition

$$
\left.\left(u \frac{\partial \phi}{\partial x}+v \frac{\partial \phi}{\partial y}-\frac{\partial}{\partial y} D \frac{\partial \phi}{\partial y}\right)\right|_{x=a}=f(a, y)
$$

It is easy to show that, in the case of an arbitrarily oriented boundary, equation (19) becomes

$$
u \frac{\partial \phi}{\partial x}+v \frac{\partial \phi}{\partial y}-\frac{\partial}{\partial s} D \frac{\partial \phi}{\partial s}=f(s) \text { for } s \in \Gamma_{\mathrm{a}}
$$

where $s$ is the coordinate tangent to the artificial boundary $\Gamma_{\mathrm{a}}$. If $u=v=0$, the boundary condition, equation $(20)$, reduces to

$$
\frac{\partial}{\partial s} D \frac{\partial \phi}{\partial s}=f(s)
$$


This equation is fully independent of the direction normal to the boundary and can be integrated in $s$. The boundary conditions are those given at the endpoints of the artificial boundary $\Gamma_{\mathrm{a}}$. Therefore, in this case, $\Gamma_{\mathrm{a}}$ becomes a boundary where a Dirichlet boundary condition is given. Depending on the boundary conditions prescribed on the rest of the boundary, the global matrix resulting from the discretization may be singular. However, this is not so in many cases, and a solution will be obtained.

As in the one-dimensional case, we illustrate the effect of the boundary conditions through a simple example. We set $D=1$ and $f(x, y)=\mathrm{e}^{-x-y}$, leaving $u$ and $v$ as parameters. The boundary conditions along $x=0$ and $y=0$ are set so that the exact solution is given by

$$
\phi(x, y)=\frac{1-\mathrm{e}^{-x-y}}{2+u+v}
$$

Along the sides $x=a$ and $y=b$, we apply the natural and convection boundary conditions, in turn.

The results show exactly the same trends as in the one-dimensional case; they improve as $L$ becomes larger and as the convective velocity normal to the artificial boundary increases. The combination of artificial boundaries along two orthogonal sides makes the case $u=v=0$ singular if the convection boundary condition is used. In Table II, we show the relative error at $(x, y)=(a, b)$ for calculations using $h=k=0 \cdot 2, L=2 \cdot 0$, and $u=v$ when the boundaries $x=a$ and $y=b$ are artificial. Either natural or convection boundary conditions are imposed on both of

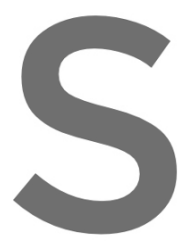
these boundaries. When a natural boundary condition is imposed on one of the boundaries and the convection boundary condition on the other, the solution falls in betveen the cases in which natural or convection boundary conditions are applied on both sides, as can be expected.

The disadvantage of the convection boundary condition as the convective velocity becomes small is evident. It should be clear that, at least in the case of convective transport equations, not much appears to be gained by using the convection condition. On the other hand, a signiffcant loss of accuracy may occur if convection is not clearly dominant over diffusion.

\section{THE NAVIER-STOKES EQUATIONS}

Of particular importance is the effect of artificial boundaries on the modelling of non-linear Navier-Stokes equations consisting of the mass conservation equation and the momentum equations for incompressible flows. The mass conservation equation is (using indicial notation for conciseness)

$$
\frac{\partial u_{i}}{\partial x_{i}}=0
$$

Table II. Relative error (in per cent) at $(x, y)=(a, b)$ in the solution of equation $(14)$ using linear elements with natural and convection boundary conditions

\begin{tabular}{lcc}
\hline$u$ & Natural & Convection \\
\hline 0.01 & 10.0 & 444.0 \\
0.1 & 9.0 & 46.0 \\
1.0 & 5.0 & 4.0 \\
10.0 & 0.8 & 0.3 \\
\hline
\end{tabular}


where, in two dimensions, $x_{1}=x, x_{2}=y, u_{1}=u$ and $u_{2}=v$. We also assume summation over repeated indices. There are two different ways to write the momentum equations, each of which produces a different set of boundary conditions at open boundaries. We will therefore examine them separately.

\subsection{Momentum equations in terms of stresses}

In terms of the stress tensor, the momentum equations take the form

$$
\rho u_{j} \frac{\partial u_{i}}{\partial x_{j}}=\frac{\partial}{\partial x_{j}} \sigma_{i j}+\rho f_{i}
$$

where $p$ is the density, $f_{i}$ are the components of the body force vector, and the components of the stress tensor for a Newtonian fluid are given by

$$
\sigma_{i j}=-p \delta_{i j}+\mu\left(\frac{\partial u_{i}}{\partial x_{j}}+\frac{\partial u_{j}}{\partial x_{i}}\right)
$$

Here, $p$ is the pressure, $\mu$ is the dynamic viscosity, and $\delta_{i j}$ is the Kronecker delta function.

The Galerkin weighted residuals form of equation (25) yields the natural boundary conditions
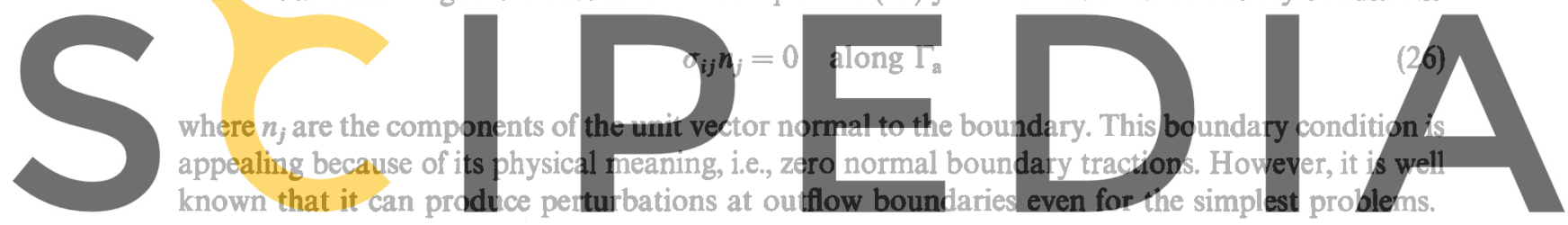

where $n_{j}$ are the components of the unit vector normal to the boundary. This boundary condition is
appealing because of its physical meaning, i.e., zaro normal boundary tractoons. However, it is yell
known that it can produce perturbations at outflow boundaries even for the simplest problems.

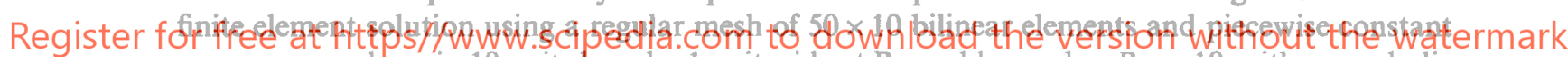
pressure on a domain 10 units long by 1 unit wide at Reynolds number $R e=10$, with a parabolic profile imposed at $x=0$. This solution clearly shows a vertical spreading of the velocity profile at the outlet. In this case, the cause of the perturbation at the boundary can be attributed to the fact that the natural boundary conditions impose a zero normal shear stress on the boundary, i.e.,

$$
\mu\left(\frac{\partial u}{\partial y}+\frac{\partial v}{\partial x}\right)=0 \quad \text { at } x=10
$$

and, because $\partial u / \partial y$ is a linear function of $y$, the vertical velocity $v$ is perturbed at the boundary. It is easy to see that the effect is magnified for low Reynolds numbers and that it tends to disappear as $R e$ increases and the viscous forces are less important.

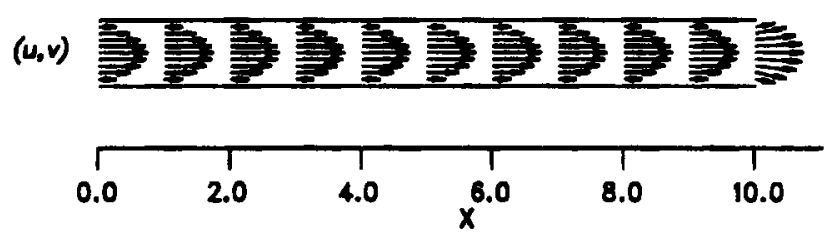

Figure 3. Isothermal plane Poiseuille flow at $R e=10$, using homogeneous natural stress conditions at $x=10$ 
The situation becomes considerably more complicated for stratified flows or, in general, for flows subjected to significant body forces. Consider the case of a Boussinesq fluid, with the body forces given by

$$
\rho f_{i}=\rho_{0}\left[1+\beta\left(T-T_{0}\right)\right] \delta_{2 i}
$$

where $\rho_{0}$ and $T_{0}$ are reference density and temperature, respectively, and $\beta$ is the coefficient of thermal expansion. The temperature, $T$, is given by the energy equation

$$
u_{i} \frac{\partial T}{\partial x_{i}}=\alpha \frac{\partial^{2} T}{\partial x_{i} \partial x_{i}}
$$

with $\alpha$ being the thermal diffusivity. To illustrate the magnitude of the perturbation introduced by the. natural stress boundary condition at an outflow boundary, we look at the problem of stratified flow over a backward facing step first solved by Leone; ${ }^{9}$ the domain and boundary conditions are shown in Figure 4. The solution obtained for Reynolds number $R e=800$, Peclet number $P e=800$, and Froude number $F r=16 / 9$ using a mesh of 6400 bilinear elements is shown in Figure 5, where it can be seen that the flow at the outlet, which should be fully developed, has been perturbed to the extent that an artificial (numerical) recirculation cell is present at the bottom. All dependent variables, velocities, temperatures, and pressures have been modified at the outlet.

Such a large perturbation is due, in this case, to the first of the natural stress boundary conditions that requires

$$
-p+2 \mu \frac{\partial u_{1}}{\partial x_{1}}=0
$$

along the boundary $x=12 \cdot 5$. Because the flow should be fully developed as it exits the computational region, equation (30) essentially attempts to impose $p=0$ along $x=12.5$. This is not possible in a stratified situation with a non-linear vertical pressure distribution unless the static component of the pressure is removed. ${ }^{9,13}$

(a)

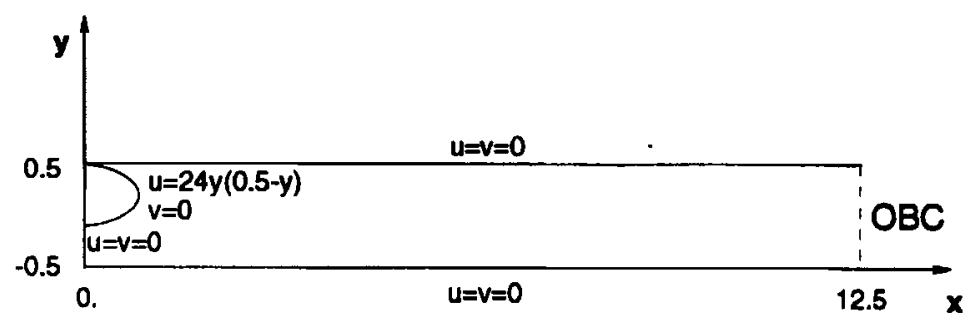

(b)

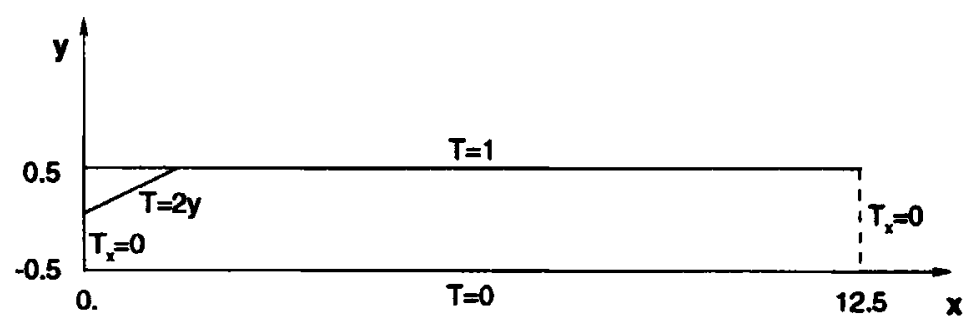

Figure 4. Domain and boundary conditions for stratified flow over a backward facing step 

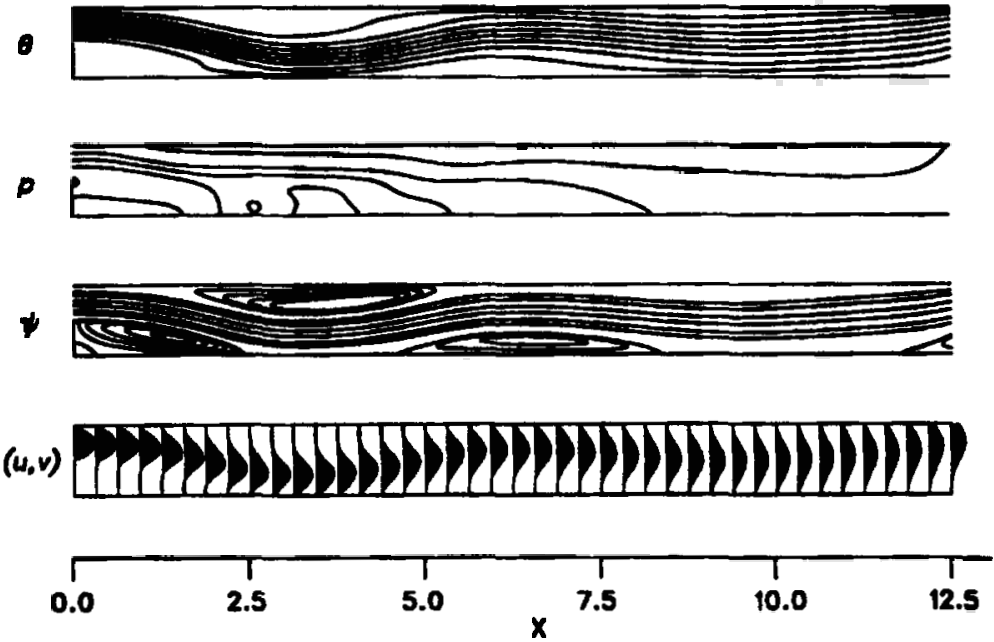

Figure 5. Results for stratified flow over a backward facing step at $R e=P e=800$ and $F r=16 / 9$, using homogeneous natural stress boundary conditions

The problem discussed above does not occur if the convection boundary condition is used. The convection boundary condition takes the form

$$
\rho u_{j} \frac{\partial u_{i}}{\partial x_{j}}-\frac{\partial}{\partial s}\left(\sigma_{i j} s_{j}\right)=\rho f_{i} \text { along } \Gamma_{\mathrm{a}}
$$

where $s_{j}$ are the components of the unit vector tangent to $\Gamma_{\mathrm{a}}$. An analysis similar to that in Section 3 shows that the component of the pressure normal to $\Gamma_{\mathrm{a}}$ is eliminated from the equations of the boundary nodes. We will discuss this further in the next section.

\subsection{Momentum equations in terms of velocities}

A substitution of the mass conservation equation into equation (24) allows us to rewrite the momentum equations in terms of the Laplacian of the velocity components, i.e.,

$$
\rho u_{j} \frac{\partial u_{j}}{\partial x_{j}}=-\frac{\partial p}{\partial x_{j}}+\mu \frac{\partial^{2} u_{j}}{\partial x_{j} \partial x_{j}}+\rho f_{i}
$$

and the natural boundary conditions take the form

$$
\left(-p \delta_{i j}+\mu \frac{\partial u_{i}}{\partial x_{j}}\right) n_{j}=0
$$

Regardless of whether the velocity or stress form of the equations is used, an analysis similar to the one presented in Section 3 for the convection diffusion equation shows that the mixed formulation, which uses the bilinear element to discretize the velocity, uses a piecewise constant pressure (or selective reduced one-point integration of the penalty term in a penalty function formulation), and retains the pressure boundary term

$$
\int_{\Gamma_{\mathrm{a}}} w p \delta_{i j} n_{j} \mathrm{~d} \Gamma
$$


removes the normal component of the pressure along the boundary nodes. Noting that the integral (34) represents the reaction at the boundary to the internal forces due to the pressure, this effectively eliminates external perturbations on the open boundary due to pressure forces external to the domain, while maintaining equilibrium of the internal forces.

Furthermore, the natural boundary condition becomes

$$
\frac{\partial u_{i}}{\partial x_{j}} n_{j}=0
$$

or zero normal velocity gradients, for the velocity form of the equations, and the slightly different form

$$
\mu\left(\frac{\partial u_{i}}{\partial x_{j}}+\frac{\partial u_{j}}{\partial x_{i}}\right) n_{j}=0
$$

when stresses are used. Considering that the pressure gradients in equations (24) and (32) are a forcing term, the results in Section 3 with regard to natural boundary conditions also apply to equations (24) and (32), with the boundary conditions (36) and (35), respectively.

We conclude that the use of natural or convection boundary conditions on the velocity components does not have a significant effect on the numerical solutions. The main source of error in the outflow boundary stems from unwittingly applied normal pressure distributions along these boundaries when the pressure term is allowed to remain a part of the natural boundary condition. The problem is eliminated if the boundary integral of the pressure, equation (34), is retained in the discretized equations. There are other ways to achieve this, such as separating the pressure into static and dynamic components in such a way that only the dynamic part is left in equations (31) and (32). This approach has also been investigated. ${ }^{14,15}$

A corrected numerical solution to the developed plane Poiseuille flow of Figure 3, calculated with the velocity form of the momentum equations, is shown in Figure 6. The interesting thing about this example is that it provides an exception to the rule, in that the pressure at the outlet boundary does not cause the incorrect velocities, as mentioned in Section 4.1. In this case, removing the pressure in equation (30) does not correct the problem which stems from the natural boundary condition on the shear stress given by equation (27).

An example of stratified flow over a backward facing step is now solved using the momentum equation (32) and natural boundary condition (35). The pressure integral (34) is incorporated into the discretization. The solution is shown in Figure 7. Clearly, the problem at the outlet has disappeared. In this case, both forms of the equations yield practically the same results, as long as the component of the pressure normal to the outflow boundary is removed. The effect of equation (27) in the stress form of the equation becomes negligible for this large Reynolds number.
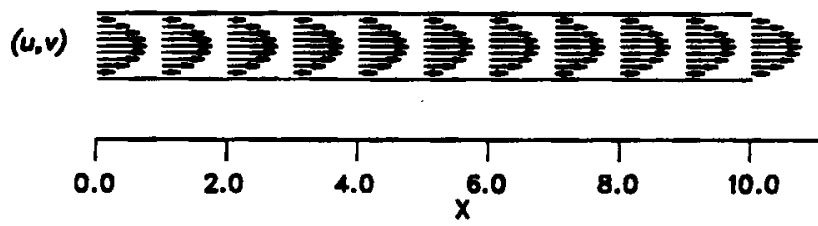

Figure 6. Results for isothermal Poiseuille flow at $R e=10$, using the natural boundary condition, equation (33), with the pressure removed from the boundary equations 
$\boldsymbol{\theta}$

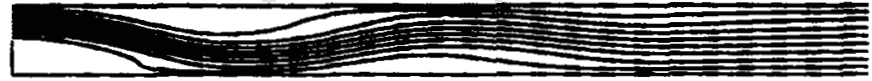

p
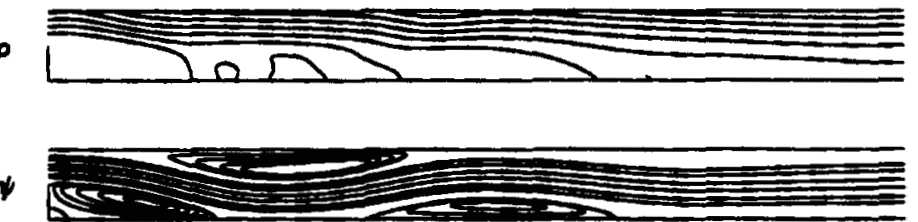

(a.) (4)

Figure 7. Results for the stratified flow over a backward facing step shown in Figure 5, but using the same outflow boundary conditions as in Figure 6

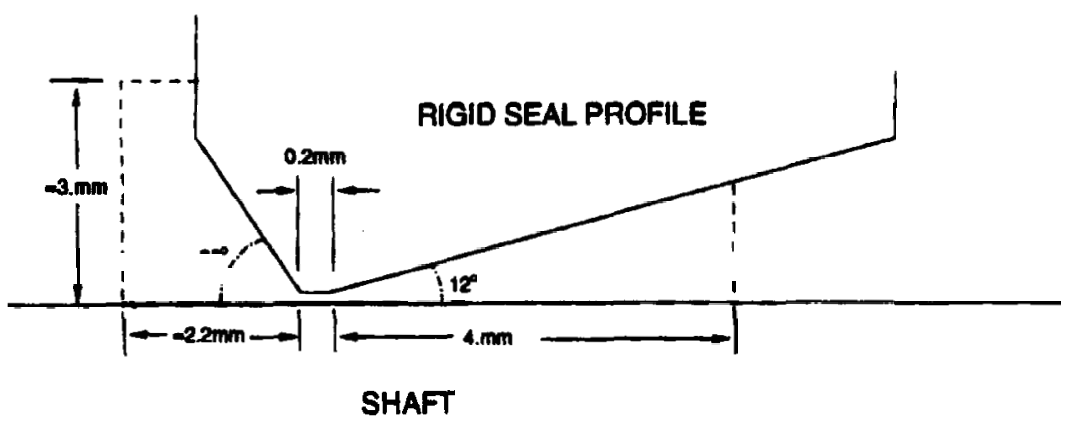

Figure 8. Schematic of the contact region between an elastomeric seal and a rotating shaft

\section{A PRACTICAL EXAMPLE}

In some cases, we need to introduce arbitrary boundaries, even if the flow fields outside the region may have some structure. We expect that these do not affect the local flows of interest. Consider axisymmetric flow through a very thin gap, representative of an elastomeric seal. Figure 8 shows the idealized axisymmetric domain. The momentum equations are written in non-dimensional form as

$$
u_{j} \frac{\partial u_{i}}{\partial x_{j}}=\frac{\partial}{\partial x_{j}} \sigma_{i j}+T a w^{2} \delta_{i 2}
$$

where $T a$ is the Taylor number and $w$ is the circumferential velocity given by the convective transport equation

$$
u_{j} \frac{\partial w}{\partial x_{j}}=\frac{\partial^{2} w}{\partial x_{j} \partial x_{j}}
$$

The body force term in equation (37) accounts for rotational inertia, which drives the flow in the $x-y$ plane.

The computational domain and the mesh of bilinear elements used in the calculations are shown in Figure 9. The characteristic length in the non-dimensionalization is the size of the gap, 


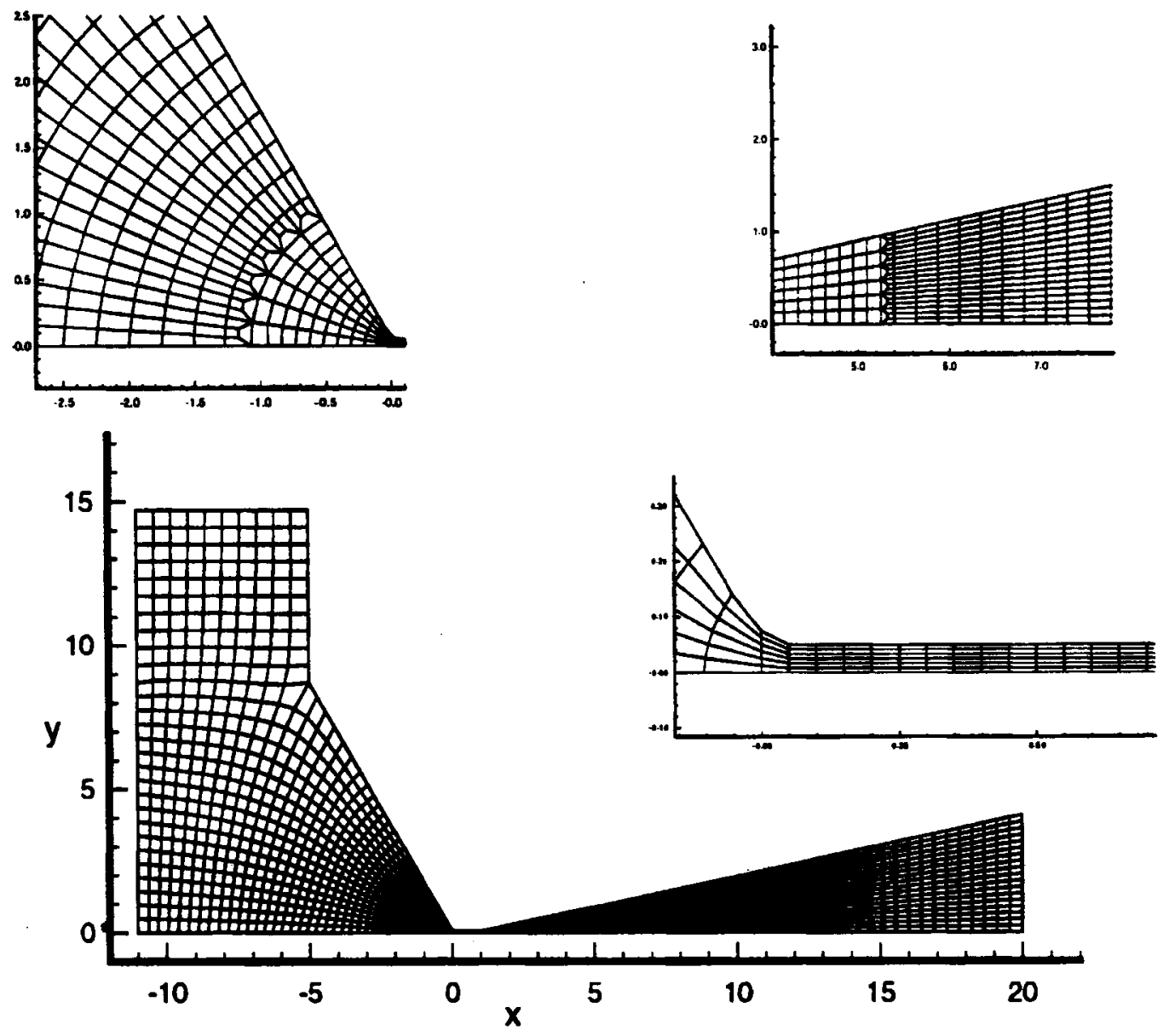

Figure 9. Domain and mesh for seal calculations

$0.2 \mathrm{~mm}$. There are three open boundaries in the domain, the left and right vertical boundaries and the top horizontal one.

Results of a numerical simulation for $T a=15$ using the natural stress boundary condition of equation (26) along the free boundaries is shown in Figure 10, where stream function contours and the flow across the gap are shown. These results are qualitatively wrong, as it is known that flow will occur from right to left, i.e., from the side with the smaller $\left(12^{\circ}\right)$ angle to the side with the larger $\left(60^{\circ}\right)$ angle.

Next, we present a solution obtained using the convection boundary condition. The other formulations with the normal component of the pressure removed at the open boundaries give very similar solutions. Figure 11 shows the results for the same Taylor number. The effect of removing the pressure from the boundary conditions is so dramatic as to reverse the direction of flow in the gap. This time, the results are at least qualitatively correct. The pressure contours are shown in Figure 12. It can be clearly seen how the pressure distribution along $x=-11.0$ is modified by the flow field when natural stress boundary conditions are used. Moreover, a strong interaction between the velocity field and the pressure takes place along the boundary $x=20.0$, 

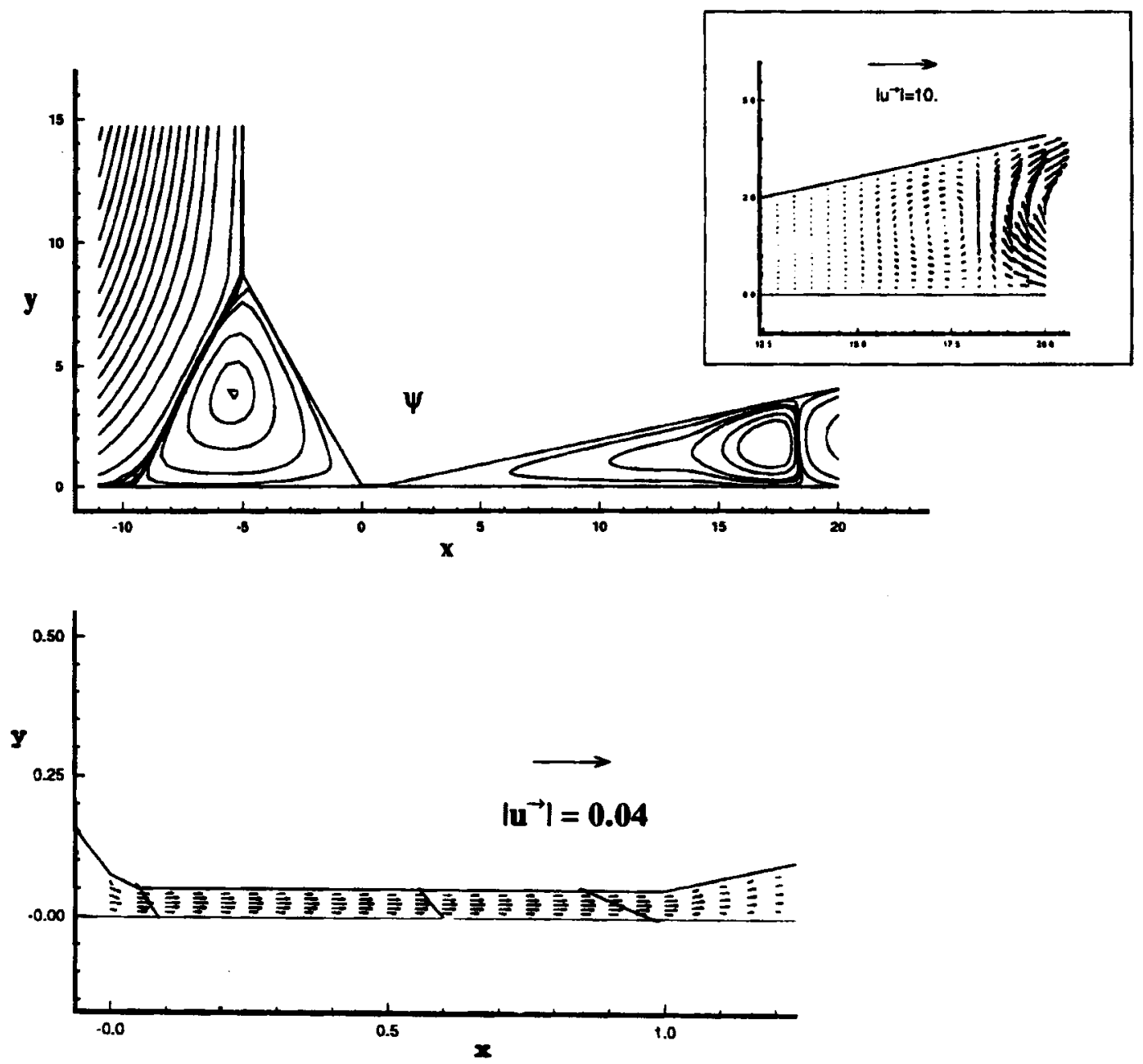

Figure 10. Calculated velocities around the seal at $T a=15$, using homogeneous natural stress boundary conditions

which introduces an artificial recirculation cell in a way similar to that of the stratified flow example shown in Figure 5. On the other hand, solutions with the pressure removed from the boundary condition show the more reasonable distribution dominated by the static component generated by the centrifugal forces.

\section{DISCUSSION AND CONCLUSIONS}

We have examined the boundary conditions normally applied at outflow open boundaries in the finite element simulation of laminar, viscous incompressible flows using bilinear elements.

For convective transport equations, either a zero diffusive flux normal to the boundary or a boundary condition of the Sommerfeld type (here we have called it the 'convection' boundary condition), seem to be the most reasonable possibilities. The convection boundary condition has the drawback that it can produce a badly conditioned problem if the convective velocities are 

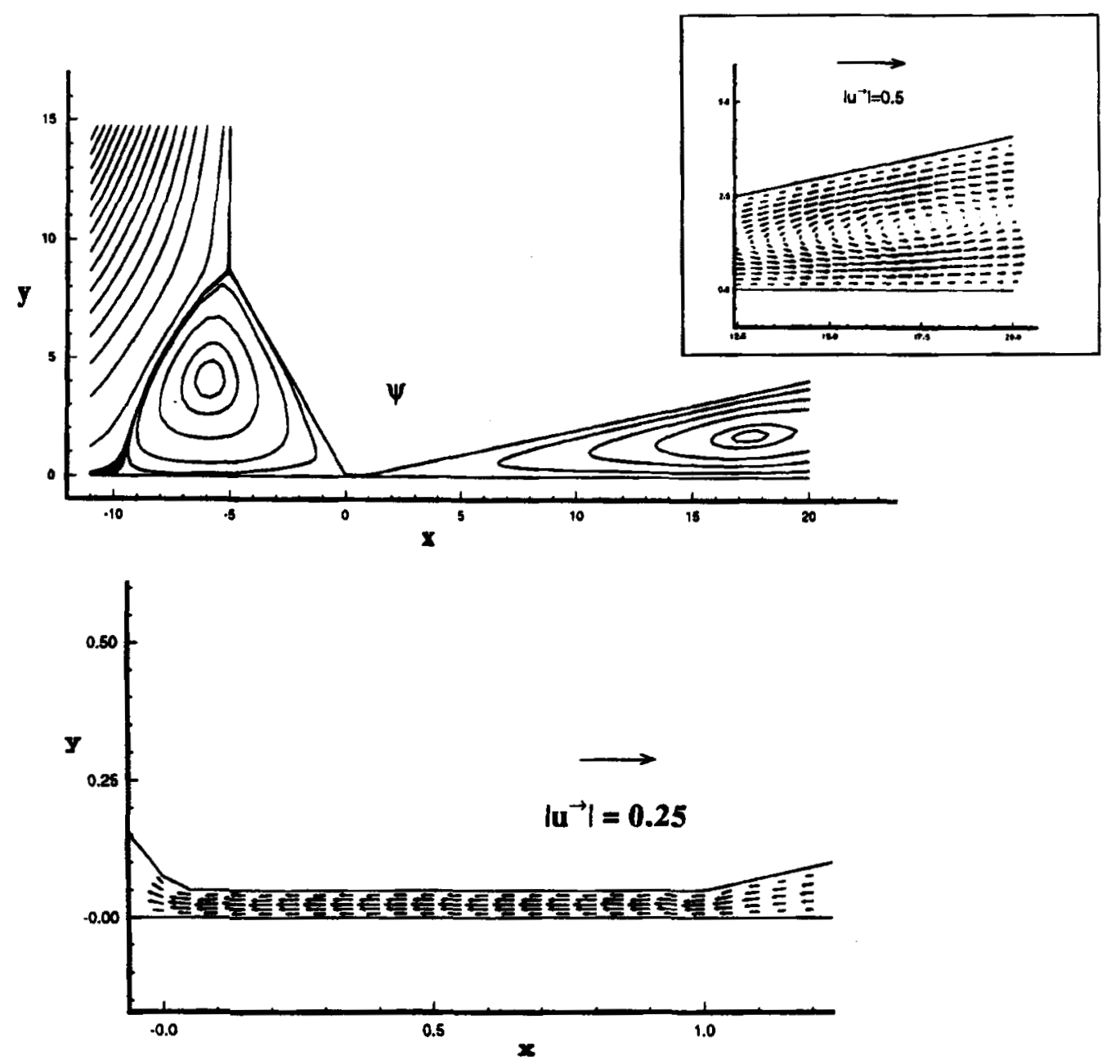

Figure 11. Calculated velocities around the seal at $T a=15$, using open boundary conditions with the pressure removed

very small and a singular one when they vanish. Homogeneous natural boundary conditions do not suffer from this problem and, in general, result in better accuracy, except when the problems are strongly dominated by convection, as can be surmised from the results presented in Tables I and II.

In general, the two boundary conditions discussed above appear to bracket the exact solution when the source function $f$ is not zero. If the solutions using both methods are reasonably close, indicating that the convection boundary condition is not producing an ill-posed problem, the average of the two approximations should provide an improved solution of greater accuracy.

For the two-dimensional Navier-Stokes equations, the key to avoiding errors due to the open boundaries lies in the removal of the pressure term from the boundary conditions. For an incompressible fluid, no boundary conditions on the pressure are required and, therefore, none should be imposed (unless, of course, the pressure is the driving force and is provided as input data at one or more open boundaries). In fact, the excellent results observed when the convection boundary condition is applied ${ }^{\mathbf{1 0 , 1 1 , 1 5}}$ are due to the fact that the pressure is eliminated from the boundary equations when the boundary integrals are retained and discretized. 


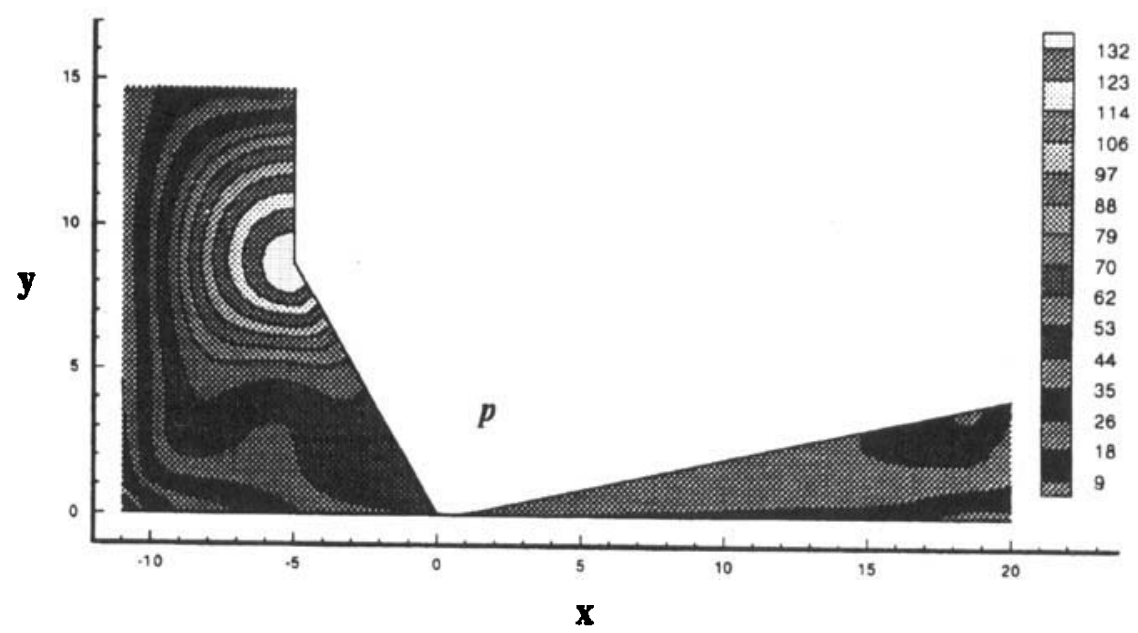

(a)

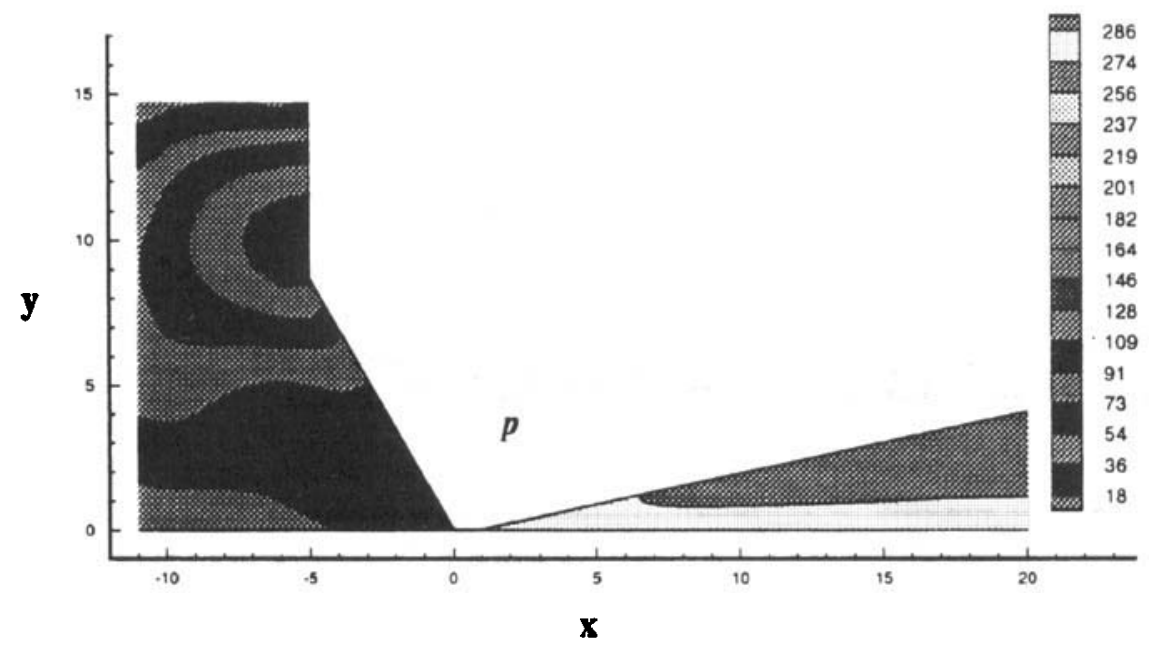

(b)

Figure 12. Pressure contours: (a) homogeneous natural stress boundary condition; (b) pressure removed from the open boundary

The results are valid in three dimensions, provided that trilinear brick elements are used. However, the results do not appear to be valid for higher-order elements. In particular, we say in Section 2.2 that the use of the quadratic elements and the convection boundary condition can result in unacceptable errors. Also, it is not difficult to show that, in the discretization of the Navier-Stokes equations using biquadratic elements, retention of the boundary integrals in the formulation does not cancel the pressure contributions exactly at boundary nodes if the pressure is assumed to be non-conforming and linear over each element. However, if the problems are strongly dominated by convection, excellent results seem to be attained both for convective diffusion equations and the Navier-Stokes equations, suggesting that the undesired terms become of secondary importance at high Reynolds numbers. 
From our numerical results, the most appropriate conditions result when the momentum equations are written in terms of velocities, equation (32); the pressure is removed from the boundary conditions by retaining the line integrals of the pressure at the open boundaries, equation (34); and the homogeneous natural boundary conditions, equation (35), are applied on the velocities. Other forms of the open boundary conditions can be found in the literature, and particular problems may be properly modelled by other methods. In general, though, the treatment of the open boundaries described above provides a consistent and robust method that has proven successful in a variety of different simulations.

\section{ACKNOWLEDGEMENTS}

The first two authors (JCH and SRI) are indebted to the Spanish Ministry of Science and Education for the support received during their sabbatical stays at the U.P.C. in Barcelona.

\section{REFERENCES}

1. R. W. Yeung, 'Numerical methods in free surface flows', Ann. Rev. Fluid Mech., 14, 395-442 (1982).

2. B. Gustaffson and H. O. Kreiss, 'Boundary conditions for time dependent problems with an artificial boundary', J. Comput. Phys., 30, 333-351 (1979).

3. K. W. Thompson, 'Time-dependent boundary conditions for hyperbolic systems, II', J. Comput. Phys., 89, 439-461 (1990).

4. M. Johnson, K. D. Paulsen, and F. E. Werner, 'Radiation boundary conditions for finite element solutions of generalized wave equations', Int. $j$. numer. methods fluids, 12, 765-783 (1991).

5. J. E. Romate, 'Absorbing boundary conditions for free surface waves', J. Comput. Phys., 99, 135-145 (1992).

6. A. J. W. Sommerfeld, Vorlesungen über Theoretische Physik III, Mechanik der Deformierbaren Medien, Leipzig, Akademie XI (1949).

7. I. Orlanski, 'A simple boundary condition for unbounded hyperbolic flows', J. Comput. Phys., 21, 251-269 (1976).

8. D. K. Gartling, 'A test problem for outflow boundary conditions-flow over a backward facing step', Int. j. numer. methods fluids, 11, 953-967 (1990).

9. J. M. Leone, 'Open boundary conditions symposium, benchmark solutions: stratified flow over a backward facing step', Int. j. numer. methods fluids, 11, 969-984 (1990).

10. T. C. Papanastasiou, N. Malamataris and K. Elwood, 'A new outflow boundary condition', Int. j. numer. methods fluids, 14, 587-608 (1992).

11. J. C. Heinrich and C. A. Vionnet, 'On boundary conditions for unbounded flows', Comm. numer. methods eng. 11, 179-185 (1995).

12. T. Y. Han, J. C. S. Meng and G. E. Innis, 'An open boundary condition for incompressible stratified flows', J. Comput. Phys., 49, 276-297 (1983).

13. R. L. Sani and P. M. Gresho, 'Rèsumé and remarks on the open boundary condition minisymposium', Int. j. numer. methods fluids, 18, 983-1008 (1994).

14. C. A. Vionnet, 'Analysis of lubricant flows within the microgap of rotary lip seals', Ph. D. Dissertation, The University of Arizona, Tucson, 1993.

15. C. A. Vionnet and J. C. Heinrich, 'Open boundary conditions for viscous flows with variable body forces', in M. Dhanbadel et al. (eds.), Advances in Finite Element Analysis in Fluid Mechanics, FED-Vol. 171, ASME, New York, 1993, pp. 39-45. 